\title{
Perinatal Respiratory Distress
}

National Cancer Institute

\section{Source}

National Cancer Institute. Perinatal Respiratory Distress. NCI Thesaurus. Code C103190.

A pathological increase in the effort and frequency or breathing movements during the perinatal period. 